\title{
Early Outcome of TKA with a Medial Pivot Fixed-bearing Prosthesis is Worse than with a PFC Mobile-bearing Prosthesis
}

\author{
Young-Hoo Kim MD, Sung-Hwan Yoon MD, \\ Jun-Shik Kim MD
}

Received: 8 November 2007/ Accepted: 3 March 2008/Published online: 9 May 2008

(C) The Author(s) 2008

\begin{abstract}
Although the design features of the Medial Pivot fixed-bearing prosthesis reportedly improve kinematics compared with TKAs using fixed-bearings, clinical improvements have not been reported. We asked whether the clinical and radiographic outcomes, ranges of motion of the knee, patient satisfaction, and complication rates would be better in knees with a Medial Pivot fixed-bearing prosthesis than in those with a PFC Sigma mobile-bearing prosthesis. We compared the results of 92 patients who had a Medial Pivot fixed-bearing prosthesis implanted in one knee and a PFC Sigma mobile-bearing prosthesis implanted in the other. There were 85 women and seven men with a mean age of 69.5 years (range, 55-81 years). The minimum followup was 2 years (mean, 2.6 years; range, 2-3 years). The patients were assessed clinically and radiographically using the rating systems of the Hospital for Special Surgery and the Knee Society at 3 months, 1 year, and annually thereafter. Contrary to expectations, we found worse early clinical outcomes, smaller ranges of knee motion, less patient satisfaction, and a higher complication rate for the Medial Pivot fixed-bearing prosthesis than for the PFC Sigma mobile-bearing prosthesis.
\end{abstract}

Each author certifies that he or she has no commercial association (eg, consultancies, stock ownership, equity interest, patent/licensing arrangements, etc) that might pose a conflict of interest in connection with the submitted article.

Each author certifies that his or her institution has approved the human protocol for this investigation, that all investigations were conducted in conformity with ethical principles of research, and that informed consent for participation in the study was obtained.

Y.-H. Kim ( $₫)$, S.-H. Yoon, J.-S. Kim

The Joint Replacement Center of Korea, Ewha Womens

University DongDaeMun Hospital, 70, ChongRo 6-Ga,

ChongRo-Gu, Seoul 110-783, Korea

e-mail: younghookim@ewha.ac.kr
Level of Evidence: Level I, therapeutic study. See the Guidelines for Authors for a complete description of levels of evidence.

\section{Introduction}

Total knee arthroplasties (TKA) using well-designed, fixedbearing prostheses have produced good long-term results $[7,13,23]$. However, problems with polyethylene wear, osteolysis, and failure in fixation have occurred with some fixed-bearing designs [5, 6, 8, 20, 27]. Mobile-bearing knee prostheses therefore were designed to provide more conforming surface shapes with reduced polyethylene contact stresses and presumably reduced wear $[1,3,4]$. Several authors have suggested a mobile bearing could minimize bone-prosthesis interface stresses of the tibial component [17, 18]. The Medial Pivot fixed-bearing prosthesis (Advance $^{\mathrm{TM}}$, Wright Medical, Arlington, TN) was developed specifically to enhance stability and reduce polyethylene wear by creating a near constant femoral component radius to reduce contact stresses and more normal knee kinematics [21, 22].

Several authors report no or negligible clinical and radiographic differences at short- to medium-term followup with several types of mobile- and fixed-bearing prostheses in the same or different patient groups [16, 26]. These studies did not include a Medial Pivot fixed-bearing prosthesis.

We examined four hypotheses: (1) the clinical and radiographic results would be at least as good in patients having a Medial Pivot fixed-bearing prosthesis implanted (Fig. 1) as results of patients having a press-fit condylar (PFC) Sigma mobile-bearing prosthesis implanted (Fig. 2); (2) the ranges of motion (ROM) of the knee with a Medial 
Fig. 1A-C (A) Anterior and (B) lateral views and $(\mathbf{C})$ the tibial bearing surface of the Medial Pivot total knee prosthesis are shown in these photographs.
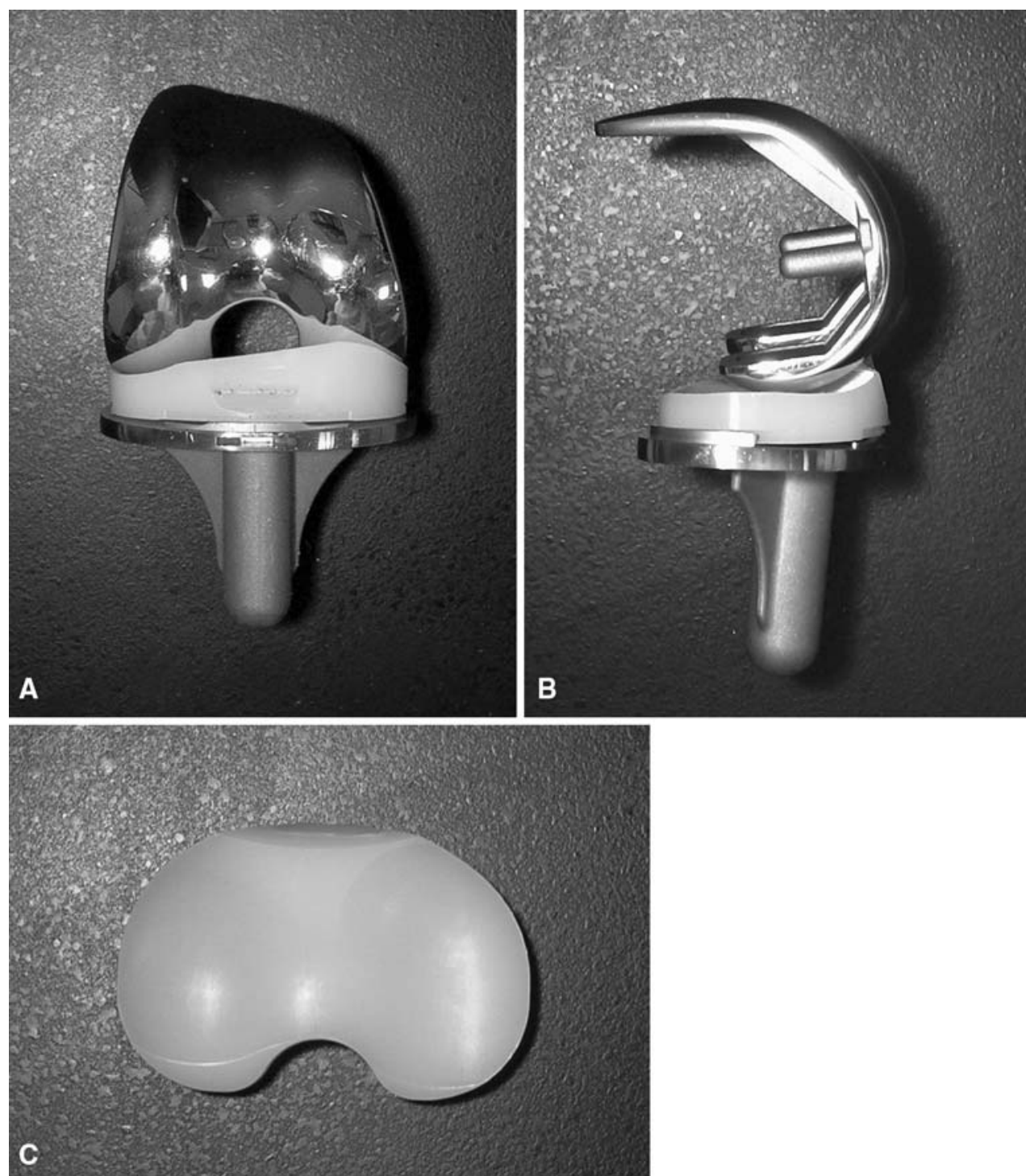

Pivot fixed-bearing prosthesis would be better than those with a PFC Sigma mobile-bearing prosthesis; (3) patient satisfaction and preference would be the same in patients having a Medial Pivot fixed-bearing prosthesis as those of patients having a PFC Sigma mobile-bearing prosthesis; and (4) complication rates would be less with the Medial Pivot fixed-bearing prosthesis than with the PFC Sigma mobile-bearing prosthesis.

\section{Materials and Methods}

We report a prospective, randomized clinical trial initiated in 2004 of a consecutive series of bilateral single-stage TKAs. All patients who had bilateral TKAs at one institution between February 2004 and February 2005 were considered for inclusion in the study. The indication for surgery was degenerative osteoarthritis that was severe enough to warrant
TKA after an adequate trial of nonoperative therapy, and the need for bilateral TKAs. We excluded patients with rheumatoid arthritis and a history of septic arthritis. Patients with rheumatoid arthritis were excluded for two reasons: (1) the clinical results between Medial Pivot fixed-bearing (Wright Medical) and PFC Sigma mobile-bearing prostheses (DePuy, Warsaw, IN) would not necessarily be comparable because of multiple joint involvement in patients with rheumatoid arthritis; and (2) progressive ligament laxity after implantation of a mobile-bearing prosthesis in patients with rheumatoid arthritis was found over a long period of time, and the knee became unstable [24]. The study protocol, including the consent forms, was approved by the Institutional Review Board at our institution. A detailed informed consent form was signed by each patient, and all information was kept confidential.

We enrolled 98 patients (198 knees) who had bilateral osteoarthritis we considered severe enough for bilateral 
Fig. 2A-C (A) Anterior and (B) lateral views and $(\mathbf{C})$ the tibial bearing surface of the PFC Sigma rotating platform prosthesis are shown in these photographs.
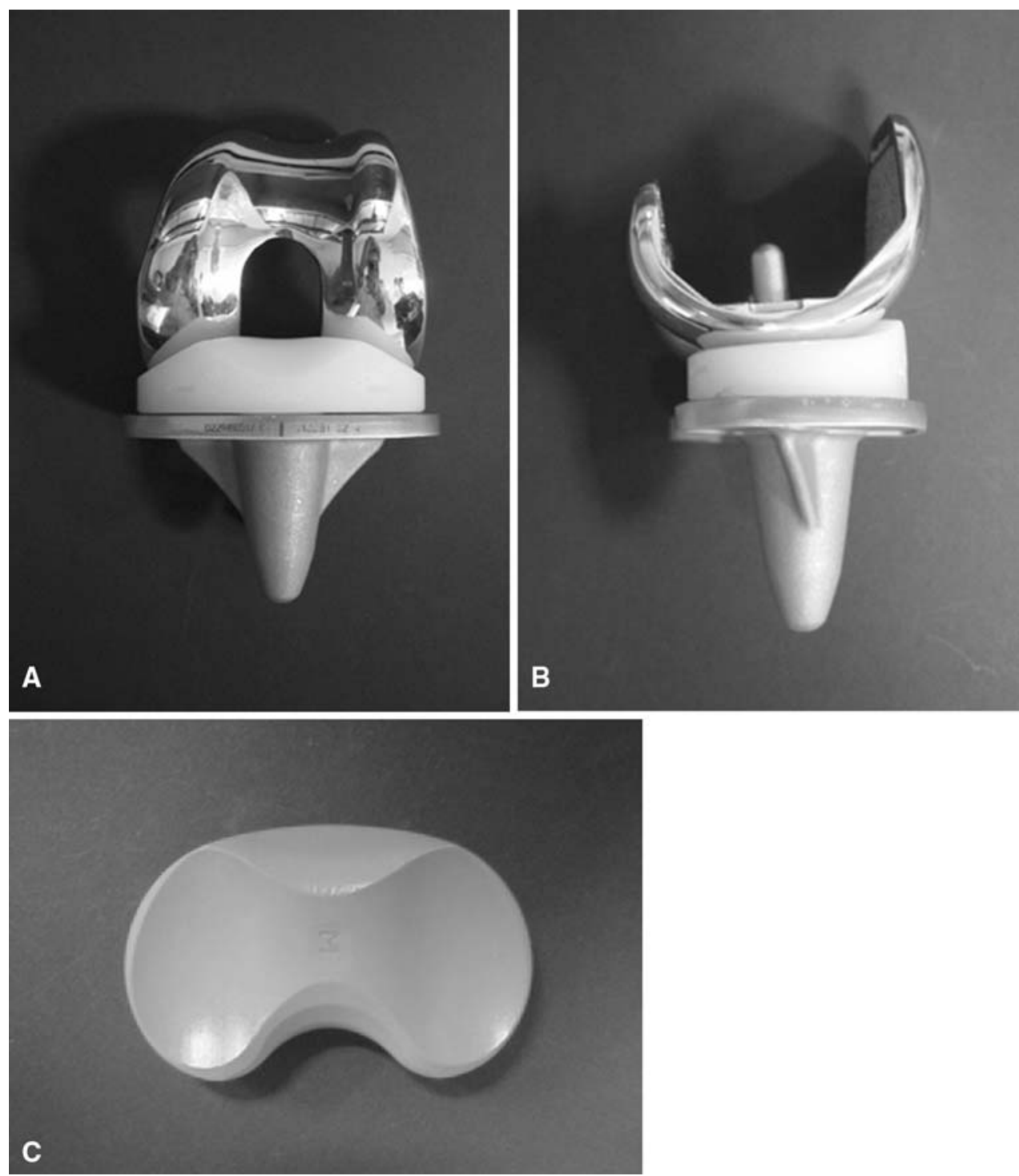

single-stage TKAs. We excluded three patients postoperatively because of subsequent diagnosis of septic arthritis, leaving 96 patients available for participation. Four patients were lost in early followup (before 3 months), leaving 92 patients (184 knees) available with a minimum followup of 2 years (mean, 2.6 years; range, 2-3 years). There were 85 female and seven male patients with a mean age of 69.5 years (range, 55-81 years; standard deviation [SD], 7.92) at the time of surgery. The high percentage of female patients in the series is attributable to this specific ethnic group of patients. All knees had a varus deformity between $8^{\circ}$ and $15^{\circ}$. Five patients with a Medial Pivot fixed-bearing TKA and six with a PFC Sigma mobilebearing TKA had previous unilateral or bilateral arthroscopic débridements and the remaining patients in both groups had no previous surgery (Table 1).
All of the included 184 TKAs in 92 patients were performed between February 2004 and April 2004. During this time, we had a high incidence of infection in the knees with the Medial Pivot fixed-bearing prostheses, and the study was stopped by the Infection Control Committee of our hospital. We investigated all possible sources of infection, including sterilization of the implant by the manufacturer and sterilization of instruments at our hospital. We found no specific factors leading to the high incidence of infection with the Medial Pivot fixed-bearing prosthesis. We resumed doing TKAs using the Medial Pivot fixed-bearing prosthesis in January 2005; 25 TKAs were performed using a Medial Pivot fixed-bearing prosthesis in one knee and a PFC Sigma mobile-bearing prosthesis in the other until the end of February 2005. Two of 25 patients had signs of infection in the knee with the Medial Pivot fixed-bearing prosthesis, and 
Table 1. Details of the 92 patients

\begin{tabular}{ll}
\hline Parameters & Mean (range; standard deviation) \\
\hline Gender (male/female) & $7 / 85$ \\
Age (years) & $69.5(55-81 ; 7.92)$ \\
Height $(\mathrm{cm})$ & $152.5(135-180 ; 7.16)$ \\
Weight $(\mathrm{kg})$ & $64.6(42-90 ; 9.18)$ \\
Body mass index $\left(\mathrm{kg} / \mathrm{m}^{2}\right)$ & $27.8(21-36 ; 3.15)$ \\
\hline
\end{tabular}

the study was discontinued permanently. A sample size estimation showed 75 knees in each group would be required to show a difference in the knee scores between the groups of at least 0.75 standard deviation from the mean with an alpha level of 0.05 and a power level of $85 \%$.

Randomization to Medial Pivot fixed-bearing TKA or PFC Sigma mobile-bearing TKA was accomplished using a sealed study number envelope, which was opened in the operating room before the skin incision was made. After opening the envelope, the first knee received the prosthesis indicated by the envelope and the contralateral (second TKA) knee received the other prosthesis. All patients underwent single-stage bilateral (sequential) TKAs under one anesthetic. Both knees were prepped and draped. The first surgery was performed on the patient's most symptomatic knee. The tourniquet was deflated after the prosthesis was implanted. Closure then was performed on the first TKA, and during this process, the surgeon communicated with the anesthesiologist to confirm the patient remained hemodynamically stable enough to undergo the second sequential TKA. The second TKA was performed in an identical manner to the first.

All surgery was performed by the senior author (YHK). A bloodless field was obtained using a pneumatic tourniquet at a pressure of $350 \mathrm{~mm} \mathrm{Hg}$ after exsanguination with an Esmarch bandage (DePuy). In all patients, an anterior midline skin incision (10-12 cm in length) was used with a medial parapatellar capsular incision of the joint. The skin incision was made as short as possible in all knees, but there were no differences in soft tissue dissection between the two groups. The degenerated anterior cruciate ligament was excised. In both groups, femoral preparation was performed first, followed by tibial preparation. Resection of the distal and posterior femoral condyles was attempted to remove a thickness of bone equal to that of the femoral component to be implanted. Although we tried to resect an equal thickness of bone from distal and posterior femoral condyles, posterolateral femoral condyles were resected less than posteromedial femoral condyles because the lateral femoral condyle is smaller than the medial femoral condyle. We attempted to set $3^{\circ}$ external rotation of the femoral component in relation to the posterior femoral condyles, or perpendicular to Whiteside's line, or parallel to the transepicondylar axis. All implants were a posterior cruciate-retaining design.

We balanced the ligaments and resected approximately $10 \mathrm{~mm}$ of tibial bone to achieve a surface that was perpendicular to the shaft of the tibia in the coronal plane with $7^{\circ}$ posterior slope in the sagittal plane. Care was taken during resection of the femur and tibia to balance the flexion and extension gaps and alleviate any flexion contracture. All patellae in both groups were resurfaced routinely using a polyethylene patellar prosthesis. The patellar thickness was measured before resection, which was equal to or slightly more than that of the component to be implanted. All implants were cemented after pulsed lavage, drying, and pressurization of cement. The status of the posterior cruciate ligament always was evaluated. At the end of implantation of the prosthesis, femoral rollback was assessed, and it was considered suboptimal when there was a liftoff and/or when the tibial articulating surface was displaced anteriorly in relation to the femoral component during $90^{\circ}$ to $120^{\circ}$ flexion of the knee.

Femoral rollback of the knee was considered suboptimal in 29 knees $(16 \%)$ with Medial Pivot fixed-bearing prostheses and in 14 knees (8\%) with PFC Sigma mobilebearing prostheses. Recession of the posterior cruciate ligament was performed in these 43 knees. We performed TKAs with the Medial Pivot prosthesis in a manner consistent with the described technique. Attention was given to checking rollback of the knee. Even if there was a subtle disturbance of rollback in the knee with a Medial Pivot prosthesis, the posterior cruciate ligament was recessed. We suspect constrained configuration of the medial compartment of the Medial Pivot fixed-bearing prosthesis might be a reason for a higher rate of posterior cruciate ligament recession. The tourniquet was deflated immediately after insertion of the components. Hemostasis was completed and the wound was closed in layers. The mean tourniquet time was 30 minutes for the Medial Pivot fixedbearing prosthesis and 35 minutes for the PFC Sigma mobile-bearing prosthesis. Additional time was spent doing more meticulous ligament balance to prevent subluxation or dislocation of the tibial bearing surface in knees with PFC Sigma mobile-bearing prostheses.

A splint was applied with the knee in $15^{\circ}$ flexion and was worn for the first 24 hours after surgery. The patients used a continuous passive motion machine for passive ROM exercises twice daily for 30 minutes each time. The settings on the machine were advanced incrementally under the supervision of the therapist until $120^{\circ}$ flexion (range, 7-10 days) was achieved. Active ROM exercise also was performed under the supervision of the therapist twice daily for 30 minutes each time. On the second postoperative day, patients began standing at the bedside or walking with crutches or a walker twice daily for 
30 minutes each time under the supervision of the therapist. The patients used crutches or a walker with full weightbearing for 6 weeks and used a cane when needed thereafter.

Clinical and radiographic evaluations were made at 3 months and 1 year after surgery and yearly thereafter. All clinical data obtained at each followup were recorded and compiled by one clinical fellow (SHY) who was not part of the operative team and who had no knowledge of the radiographic findings. Preoperative and postoperative ratings according to the Knee Society [12] and Hospital for Special Surgery [14] systems were obtained for all patients. Ranges of knee motion were measured in the flesh using a goniometer during the examination period. The preoperative Knee Society knee and functional scores for knees with Medial Pivot fixed-bearing prostheses (29 and 45 points, respectively) and PFC Sigma mobile-bearing prostheses (28 and 45 points, respectively) were similar ( $\mathrm{p}=0.671$ and 1.000, respectively). Preoperative Hospital for Special Surgery knee scores for knees with Medial Pivot fixed-bearing prostheses and PFC Sigma mobilebearing prostheses (58 and 58 points, respectively) also were similar ( $\mathrm{p}=0$.699) (Table 2).

Patient satisfaction was assessed with the visual analog scale. The visual analog scale responses were grouped into four categories to determine patient satisfaction: 2 or less, fully dissatisfied; 3 to 5 , somewhat dissatisfied; 6 to 8 , satisfied; and 8 to 10 , fully satisfied. This method was described previously [3]. It is somewhat arbitrary and has not been scientifically validated, but nevertheless, we believe it provides a useful impression of the degree of patient satisfaction.

All 92 patients had complete radiographic followups. Radiographs were analyzed by one observer (JSK) who had no knowledge of the names of the patients to determine consistency in radiographic assessment, and the findings were recorded by a research assistant (SML) who knew the names of the patients. Radiographs obtained before and after surgery, which included anteroposterior (AP) radiographs obtained with the patient standing and supine, a lateral radiograph, and a skyline patellar radiograph, were assessed for alignment of the limb, position of the component, and presence and location of all radiolucent lines at the bone-cement interface according to the recommendation of the Knee Society [12]. All radiographs were taken under the fluoroscopic control to ensure adequate observation of the interfaces. The joint lines were determined on AP radiographs obtained before and after surgery with the patient supine by measuring the distance between the tip of the fibular head and the distal margin of the lateral femoral condyle preoperatively and between the tip of the fibular head and the distal margin of the lateral femoral component

Table 2. Preoperative data

\begin{tabular}{|c|c|c|c|c|}
\hline \multirow[t]{2}{*}{ Parameters } & \multicolumn{2}{|c|}{ Knee Society score } & \multicolumn{2}{|c|}{ Hospital for Special Surgery knee score } \\
\hline & Medial Pivot & PFC Sigma & Medial Pivot & PFC Sigma \\
\hline Total knee score (points) & $29(2-50)$ & $28(0-50)$ & $58(45-70)$ & $58(38-70)$ \\
\hline Functional score (points) & $45(20-60)$ & $45(20-60)$ & - & - \\
\hline Pain score (points) & 0 & 0 & $8(0-15)$ & $8(0-15)$ \\
\hline None & - & - & - & - \\
\hline Mild & - & - & - & - \\
\hline Moderate & - & - & - & - \\
\hline Severe & $92(100 \%)$ & $92(100 \%)$ & $92(100 \%)$ & $92(100 \%)$ \\
\hline \multicolumn{5}{|l|}{ Walking distance } \\
\hline Cannot walk & \multicolumn{2}{|c|}{1 patient $(1 \%)$} & \multicolumn{2}{|c|}{1 patient $(1 \%)$} \\
\hline Less than one block & \multicolumn{2}{|c|}{90 patients $(98 \%)$} & \multicolumn{2}{|c|}{90 patients $(98 \%)$} \\
\hline $1-5$ blocks & \multicolumn{2}{|c|}{1 patient $(1 \%)$} & \multicolumn{2}{|c|}{1 patient $(1 \%)$} \\
\hline Range of motion (degrees) & $124(60-150)$ & $124(50-150)$ & $124(60-150)$ & $124(50-150)$ \\
\hline \multicolumn{5}{|l|}{ Walking support } \\
\hline No support & \multicolumn{2}{|c|}{15 patients $(15 \%)$} & \multicolumn{2}{|c|}{15 patients $(15 \%)$} \\
\hline One cane & \multicolumn{2}{|c|}{73 patients $(80 \%)$} & \multicolumn{2}{|c|}{73 patients $(80 \%)$} \\
\hline One crutch & \multicolumn{2}{|c|}{1 patient $(1 \%)$} & \multicolumn{2}{|c|}{1 patient $(1 \%)$} \\
\hline Two crutches & \multicolumn{2}{|c|}{3 patients $(3 \%)$} & \multicolumn{2}{|c|}{3 patients $(3 \%)$} \\
\hline \multicolumn{5}{|l|}{ Stairs } \\
\hline \multicolumn{5}{|l|}{ Normal } \\
\hline With support & \multicolumn{2}{|c|}{92 patients $(100 \%)$} & \multicolumn{2}{|c|}{92 patients $(100 \%)$} \\
\hline
\end{tabular}


postoperatively. The skyline patellar radiographs were examined for patellar tilt, subluxation, or dislocation. Any osteolysis around three components was recorded. We used the chance-corrected kappa coefficient to determine intraobserver agreement of radiographic measurements. Intraobserver agreement in the series was $0.61-0.80$.

We calculated descriptive statistics (means, standard deviation, and proportions) for continuous study variables. Knee Society and Hospital for Special Surgery knee scores were the primary outcome variables. These variables were analyzed with a two-way repeated measures analysis of variance. Ranges of motion of the knee also were compared between the two groups using a two-way repeated measures analysis of variance. Complication rates and radiographic data were compared between the two groups with nonparametric chi square tests. All statistical analyses were performed with the Statistical Package for the Social Sciences (SPSS), version 14.0 (SPSS, Chicago, IL) with two-tailed tests.

\section{Results}

The early Knee Society and Hospital for Special Surgery knee scores, pain scores, and functional scores were worse for knees with the Medial Pivot fixed-bearing than scores for knees with the PFC Sigma mobile-bearing prosthesis. However, radiographic results were similar. The postoperative Knee Society and Hospital for Special Surgery knee scores for all 92 patients were lower $(\mathrm{p}=0.021$ and $\mathrm{p}=0.023$ ) with the Medial Pivot fixed-bearing prosthesis than scores with the PFC Sigma mobile-bearing prosthesis at each followup (Tables 3,4). The postoperative Knee Society and Hospital for Special Surgery knee scores in the 81 patients who had no infection also were less $(\mathrm{p}=0.010$ and $\mathrm{p}=0.033$, respectively) with the Medial Pivot fixed-bearing prosthesis (87 and 80 points, respectively) than with the PFC Sigma mobile-bearing prosthesis (94 and 86 points, respectively). The pain score with the Medial Pivot fixed-bearing prosthesis was considerably less (patients had more pain) at the final followup than with the PFC Sigma mobile-bearing prosthesis (patients had less pain) according to both knee scoring systems $(\mathrm{p}=0.043$ and $\mathrm{p}=0.040$, respectively). No radiographic differences were observed between prostheses (Table 5) for alignment of the knee, position of the components, posterior condylar offset, and prevalence of radiolucent lines (Fig. 3). No knee in either group was revised.

Table 3. Clinical results at final followup

\begin{tabular}{|c|c|c|c|c|}
\hline \multirow[t]{2}{*}{ Parameters } & \multicolumn{2}{|l|}{ Knee Society score } & \multicolumn{2}{|c|}{ Hospital for Special Surgery knee score } \\
\hline & Medial Pivot & $\mathrm{PFC}$ & Medial Pivot & PFC \\
\hline Total knee score (points) & $87(70-100)$ & $94(80-100)$ & $87(72-98)$ & $93(73-100)$ \\
\hline Functional score (points) & $80(30-100)$ & $86(30-100)$ & - & - \\
\hline Mean pain score (points) & $35(20-50)$ points & $45(30-50)$ points & $23(10-30)$ points & $28(20-30)$ points \\
\hline None & 38 patients $(63 \%)$ & 64 patients $(70 \%)$ & 38 patients $(63 \%)$ & 64 patients $(70 \%)$ \\
\hline Mild & 51 patients $(34 \%)$ & 28 patients $(30 \%)$ & 51 patients $(34 \%)$ & 28 patients $(30 \%)$ \\
\hline Moderate & 3 patients $(3 \%)$ & - & 3 patients $(3 \%)$ & - \\
\hline Severe & - & - & - & - \\
\hline \multicolumn{5}{|l|}{ Walking distance } \\
\hline Cannot walk & \multicolumn{2}{|c|}{ - } & \multicolumn{2}{|c|}{ - } \\
\hline Less than one block & \multicolumn{2}{|c|}{ - } & \multicolumn{2}{|c|}{ - } \\
\hline $1-5$ blocks & \multicolumn{2}{|c|}{9 patients $(10 \%)$} & \multicolumn{2}{|c|}{9 patients $(10 \%)$} \\
\hline $5-10$ blocks & \multicolumn{2}{|c|}{13 patients $(14 \%)$} & \multicolumn{2}{|c|}{13 patients $(14 \%)$} \\
\hline Unlimited & \multicolumn{2}{|c|}{70 patients $(76 \%)$} & \multicolumn{2}{|c|}{70 patients $(76 \%)$} \\
\hline Range of motion (degrees) & $115(80-145)$ & $127(85-145)$ & $115(80-145)$ & $127(85-145)$ \\
\hline \multicolumn{5}{|l|}{ Walking support } \\
\hline No support & \multicolumn{2}{|c|}{84 patients $(91 \%)$} & \multicolumn{2}{|c|}{84 patients $(91 \%)$} \\
\hline One cane & \multicolumn{2}{|c|}{6 patients $(7 \%)$} & \multicolumn{2}{|c|}{6 patients $(7 \%)$} \\
\hline One crutch & \multicolumn{2}{|c|}{-} & \multicolumn{2}{|c|}{ - } \\
\hline Two crutches & \multicolumn{2}{|c|}{2 patients $(2 \%)$} & \multicolumn{2}{|c|}{2 patients $(2 \%)$} \\
\hline \multicolumn{5}{|l|}{ Stairs } \\
\hline Normal & \multicolumn{2}{|c|}{36 patients $(39 \%)$} & \multicolumn{2}{|c|}{36 patients $(39 \%)$} \\
\hline With support & \multicolumn{2}{|c|}{56 patients $(61 \%)$} & \multicolumn{2}{|c|}{56 patients $(61 \%)$} \\
\hline
\end{tabular}


Table 4. Clinical results at each followup

\begin{tabular}{|c|c|c|c|c|c|c|}
\hline \multirow[t]{2}{*}{ Parameters } & \multicolumn{3}{|c|}{ Knee Society score } & \multicolumn{3}{|c|}{ Hospital for Special Surgery knee score } \\
\hline & Medial Pivot & $\mathrm{PFC}$ & $\mathrm{p}$ Value & Medial Pivot & PFC & $\mathrm{p}$ Value \\
\hline \multicolumn{7}{|l|}{ Total knee score (points) } \\
\hline Postoperative 3 months & $79(65-91)$ & $86(75-100)$ & $<0.05$ & $80(70-89)$ & $85(75-95)$ & $<0.05$ \\
\hline 1 year & $87(70-100)$ & $94(80-100)$ & $<0.05$ & $86(72-100)$ & $93(70-100)$ & $<0.05$ \\
\hline 2.6 years & $87(70-100)$ & $94(80-100)$ & $<0.05$ & $87(72-98)$ & $93(73-100)$ & $<0.05$ \\
\hline \multicolumn{7}{|l|}{ Functional score (points) } \\
\hline Postoperative 3 months & $67(30-100)$ & $67(30-100)$ & 0.806 & - & - & - \\
\hline 1 year & $80(45-100)$ & $85(50-100)$ & 0.103 & - & - & - \\
\hline 2.6 years & $80(30-100)$ & $86(30-100)$ & 0.065 & - & - & - \\
\hline \multicolumn{7}{|l|}{ Range of motion (degrees) } \\
\hline Postoperative 3 months & $98(80-125)$ & $126(85-150)$ & $<0.05$ & - & - & - \\
\hline 1 year & $110(80-130)$ & $128(90-150)$ & $<0.05$ & - & - & - \\
\hline 2.6 years & $115(80-145)$ & $127(85-145)$ & $<0.05$ & - & - & - \\
\hline
\end{tabular}

Table 5. Radiographic results

\begin{tabular}{|c|c|c|c|}
\hline Parameters & Medial Pivot & $\mathrm{PFC}$ & $\mathrm{p}$ Value \\
\hline \multicolumn{4}{|l|}{ Alignment $\left(^{\circ}\right)$} \\
\hline Preoperative & $5^{\circ}$ varus $\left(1^{\circ}-14^{\circ}\right.$ varus $)$ & $6^{\circ}$ varus $\left(2^{\circ}-16^{\circ}\right.$ varus $)$ & 0.37 \\
\hline Postoperative & $5^{\circ}$ valgus $\left(0^{\circ}-8^{\circ}\right.$ valgus $)$ & $6^{\circ}$ valgus $\left(0^{\circ}-7^{\circ}\right.$ valgus $)$ & 0.20 \\
\hline \multicolumn{4}{|c|}{ Femoral component position (femoral angle) } \\
\hline Anteroposterior & $96^{\circ}\left(91^{\circ}-101^{\circ}\right)$ & $97^{\circ}\left(90^{\circ}-101^{\circ}\right)$ & 0.12 \\
\hline Sagittal & $3^{\circ}\left(-2^{\circ}-8^{\circ}\right)$ & $2^{\circ}\left(-3^{\circ}-6^{\circ}\right)$ & 0.21 \\
\hline \multicolumn{4}{|c|}{ Tibial component position (tibial angle) } \\
\hline Anteroposterior & $89^{\circ}\left(80^{\circ}-98^{\circ}\right)$ & $89^{\circ}\left(83^{\circ}-95^{\circ}\right)$ & 0.55 \\
\hline Sagittal & $84^{\circ}\left(77^{\circ}-95^{\circ}\right)$ & $85^{\circ}\left(77^{\circ}-91^{\circ}\right)$ & 0.17 \\
\hline Patellar component angle & $4^{\circ}\left(-13^{\circ}-26^{\circ}\right)$ & $3^{\circ}\left(-18^{\circ}-20^{\circ}\right)$ & 0.11 \\
\hline \multicolumn{4}{|l|}{ Joint line (mm) } \\
\hline Preoperative & $15.7(9-23)$ & $15.8(7-23)$ & 0.82 \\
\hline Final followup & $14.1(7-24)$ & $14.5(6-23)$ & 0.49 \\
\hline \multicolumn{4}{|c|}{ Posterior condylar offset (mm) } \\
\hline Preoperative & $23.6(18-30)$ & $23.3(18-34)$ & 0.42 \\
\hline Final followup & $23.2(19-29)$ & $23.8(18-29)$ & 0.11 \\
\hline \multicolumn{4}{|l|}{ Radiolucent line (overall) } \\
\hline Absence & 84 knees $(91 \%)$ & 87 knees $(95 \%)$ & 0.388 \\
\hline Presence & 8 knees $(9 \%)$ & 5 knees $(5 \%)$ & \\
\hline \multicolumn{4}{|c|}{ Radiolucent line (femoral side) } \\
\hline Zone 1 & 2 knees $(2 \%)$ & 1 knee $(1 \%)$ & - \\
\hline \multicolumn{4}{|l|}{ Radiolucent line (tibial side) } \\
\hline Zone 1 & 6 knees $(7 \%)$ & 4 knees $(4 \%)$ & - \\
\hline Lateral patellar tilt & 10 knees $(11 \%)$ & 7 knees $(8 \%)$ & - \\
\hline
\end{tabular}

The mean ranges of motion of the knee at the final followup were less $(p=0.713)$ with the Medial Pivot fixedbearing prosthesis compared with the PFC Sigma mobilebearing prosthesis despite mean preoperative ranges being similar $\left(124^{\circ}\right.$ versus $\left.123^{\circ}\right)$. The mean ROM at the final followup was less with the Medial Pivot fixed-bearing prosthesis than with the PFC Sigma mobile-bearing prosthesis. The mean motion at final followup was lower ( $\mathrm{p}=0.007)$ for the Medial Pivot fixed-bearing prosthesis than for the PFC Sigma mobile-bearing prosthesis 
Fig. 3A-C Radiographs show both knees of a 69-year-old woman with osteoarthritis. (A) A standing AP view of both knees obtained 3 years after surgery shows the Medial Pivot (right knee) and the PFC Sigma (left knee) prostheses are embedded solidly and satisfactorily. There are no radiolucent lines or osteolysis around the tibial components. Lateral views obtained 3 years after surgery show the (B) Medial Pivot (right knee) and the (C) PFC Sigma (left knee) prostheses appear satisfactorily fixed. There are no radiolucent lines or osteolysis around the femoral, tibial, or patellar components in either knee.
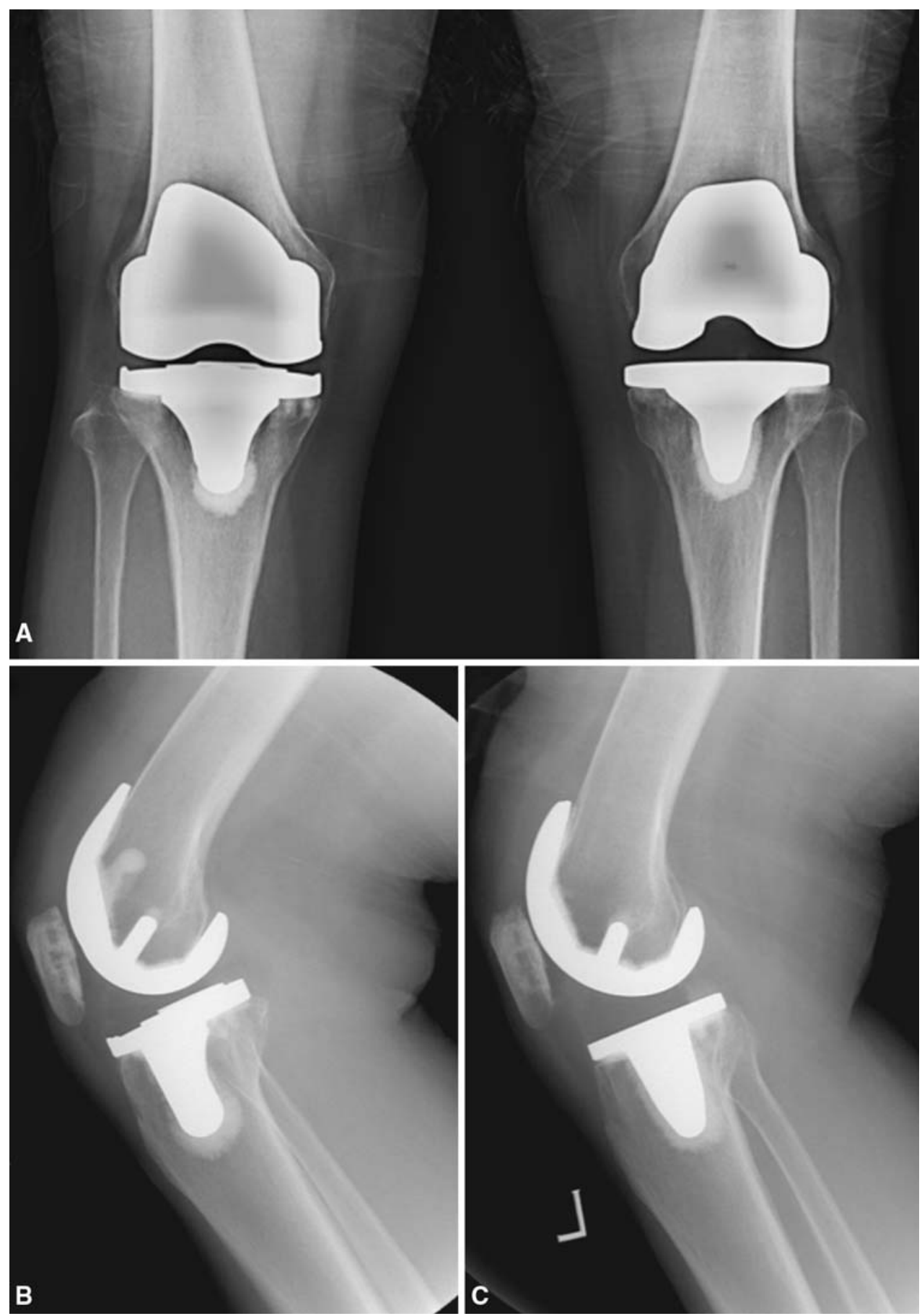

(mean $115^{\circ} \pm, 18.0^{\circ}$, versus $127^{\circ} \pm 12.1^{\circ}$, respectively) (Tables 3,4). Although the mean ROM at the final followup was decreased $(\mathrm{p}=0.001)$ in knees with the Medial Pivot fixed-bearing prosthesis, it was slightly increased $(\mathrm{p}=0.078)$ in knees with the PFC Sigma prosthesis compared with preoperative ranges (Table 2).

More patients were satisfied with the result of the TKA with the PFC Sigma mobile-bearing prosthesis than were satisfied with the result of the contralateral TKA with the Medial Pivot fixed-bearing prosthesis (Table 6). The mean satisfaction of patients with a Medial Pivot fixed-bearing prosthesis was $6.5 \pm 3.4$ points versus $7.9 \pm 2.1$ points for the PFC Sigma mobile-bearing prosthesis. Fifty-six patients $(61 \%)$ preferred the PFC Sigma mobile-bearing prosthesis; 30 patients $(33 \%)$ had no preference; and six patients $(7 \%)$ preferred the Medial Pivot fixed-bearing prosthesis.

Complication rates were greater $(\mathrm{p}=0.001)$ with the Medial Pivot fixed-bearing prosthesis than with the PFC Sigma mobile-bearing prosthesis (Table 7). Five knees 
Table 6. Patient satisfaction and preference

\begin{tabular}{lll}
\hline Parameters & Medial Pivot & PFC Sigma \\
\hline Fully satisfied & 37 patients $(40 \%)$ & 37 patients $(40 \%)$ \\
Satisfied & 31 patients $(34 \%)$ & 37 patients $(40 \%)$ \\
Somewhat dissatisfied & 13 patients $(14 \%)$ & 37 patients $(40 \%)$ \\
Fully dissatisfied & 11 patients (12\%) & 37 patients $(40 \%)$ \\
$\begin{array}{l}\text { Causes of dissatisfaction } \\
\quad \text { Infection }\end{array}$ & 11 of 24 patients & 1 of 6 patients \\
$\quad \begin{array}{l}\text { Flexion contracture } \\
\text { Recurrent effusion }\end{array}$ & 3 of 24 patients & - \\
$\quad \begin{array}{l}\text { Insufficient range } \\
\text { of motion of knee }\end{array}$ & 1 of 24 patients & - \\
$\begin{array}{c}\text { Constant mild pain } \\
\text { and stiffness }\end{array}$ & - & 4 of 6 patients \\
\hline
\end{tabular}

Table 7. Complications

\begin{tabular}{lcl}
\hline Parameters & $\begin{array}{c}\text { TKA with Medial } \\
\text { Pivot prosthesis }\end{array}$ & $\begin{array}{l}\text { TKA with PFC } \\
\text { Sigma prosthesis }\end{array}$ \\
\hline $\begin{array}{l}\text { Deep infection* } \\
\text { Superficial infection }{ }^{\dagger}\end{array}$ & 5 knees $(2.7 \%)$ & 1 knee $(0.5 \%)$ \\
$\begin{array}{l}\text { Supracondylar fracture } \\
\text { (open reduction }\end{array}$ & 1 knee $(0.5 \%)$ & - \\
$\quad$ and internal fixation) &
\end{tabular}

with the Medial Pivot fixed-bearing prosthesis had early deep infections (coagulase negative Staphylococcus epidermidis) and one knee with the PFC Sigma mobilebearing prosthesis had an early deep infection (coagulase positive Staphylococcus aureus). The patients were treated with open débridement of the knee followed by intravenous antibiotics for 6 weeks. No patient had recurrence of deep infection. Six patients $(6.5 \%)$ with superficial infections in knees with the Medial Pivot fixed-bearing prosthesis were treated with intravenous antibiotics only for 2 to 4 weeks. There was no recurrence of infection.

In the knees with a Medial Pivot fixed-bearing prosthesis, three $(2 \%)$ had a persistent flexion contracture, nine $(5 \%)$ had a recurrent effusion, and one $(0.5 \%)$ had skin edge necrosis. One patient (one knee; 0.5\%) sustained a supracondylar fracture after a fall. Open reduction and internal fixation was performed augmented with a freshfrozen femoral shaft allograft. In the knees with a PFC Sigma mobile-bearing prosthesis, a deep peroneal nerve palsy developed in one knee $(0.5 \%)$. This resolved completely within 1 year of surgery. There were no bearingrelated complications in the patients with a PFC Sigma mobile-bearing prosthesis such as bearing subluxation, dislocation, or failure.

\section{Discussion}

Although the design features of the Medial Pivot fixedbearing prosthesis reportedly improve kinematics compared with previous fixed-bearing prostheses, this has not been documented clinically. To compare the results of the Medial Pivot fixed-bearing and PFC sigma mobile-bearing prostheses, we asked whether the clinical and radiographic results, ROM of the knee, patient satisfaction, and complication rates would be better in knees with a Medial Pivot fixed-bearing prosthesis than in knees with a PFC Sigma mobile-bearing prosthesis.

There are some limitations of this study. First, because early cessation of this study related to the high infection rate, our study is underpowered to make any conclusions regarding our primary outcome [11]. Second, we have no interobserver variability and this can lead to substantial bias in interpreting radiolucent lines and loosening. Third, it is difficult for a patient who has undergone bilateral TKAs to separate the function of each knee. Although this is a problem when assessing function after bilateral TKAs, we believe we were able to obtain fairly accurate information after careful assessment of the performance of each knee. Comparing the benefits of two different treatments in the same patients has the advantage that patient-dependent prognostic factors are eliminated, although it introduces the problem with the difficulty of a patient separating function of the two knees, particularly the overall function. However, our comparisons are within-patient (paired) and not between patients as is more typical. The advantages are that fewer patients are required and confounding factors are controlled. These problems still occur in traditional parallel group trials despite efforts to minimize the potential for bias. Finally, the followup was short and we can make no conclusions regarding the theoretical advantage of the Medial Pivot fixed-bearing prosthesis regarding wear.

Numerous prosthetic designs now are available, including mobile- and fixed-bearing designs. However, few authors provide direct comparisons of outcomes of any two or more types of prostheses. The results of AMK fixed-bearing (DePuy) and LCS meniscal-bearing (DePuy) prostheses 
were prospectively compared in 222 patients (444 knees) who had bilateral simultaneous TKAs [16]. At mean followups of 7.4 and 10.3 years, respectively, the authors reported no differences in clinical outcomes between the two groups. The Nexgen legacy posterior stabilized (LPS) mobile-bearing and fixed-bearing prostheses (Zimmer, Warsaw, IN) have been compared and again no major clinical advantages were found for the mobile-bearing design [26]. The PFC Sigma mobile-bearing and PFC Sigma fixedbearing prostheses (DePuy) were compared in 174 patients (348 knees) with a mean followup of 5.6 years [15]. Again, no difference in clinical outcome was identified between the two groups.

We found the early clinical outcomes of knees with the Medial Pivot fixed-bearing prostheses were worse than those of knees with PFC Sigma mobile-bearing prostheses. The postoperative Knee Society and Hospital for Special Surgery knee scores in patients who had no infection were lower for those with the Medial Pivot fixed-bearing prosthesis (87 and 86 points, respectively) than for patients with the Sigma prosthesis (94 and 93 points, respectively). Therefore, infection was not an effecter modifier to influence the outcomes so that patients with the Medial Pivot fixed-bearing prosthesis had worse outcomes. We are uncertain why this particular Medial Pivot fixed-bearing prosthesis had worse shortterm clinical outcomes. We suspect the excessive constraint of the Medial Pivot fixed-bearing prosthesis imposed by the fully congruent medial tibiofemoral articulation may not restore normal kinematics of the knee and inhibit posterior rolling and sliding of the lateral femoral condyle around a medial femoral condyle during knee flexion. This subtle disturbance of kinematics of the Medial Pivot fixed-bearing prosthesis may contribute to less favorable clinical outcomes than the PFC Sigma mobile-bearing prosthesis.

Numerous authors have compared ROM in knees with fixed- and mobile-bearing prostheses [15, 16, 25, 26]. These authors reported no or negligible differences between the two types of implants. In one study, specifically, the ranges of motion of the knee were compared between the PFC Sigma fixed-bearing and PFC Sigma mobile-bearing prostheses [15]. No difference in the ranges was identified between the two groups $\left(131^{\circ}\right.$ and $\left.130^{\circ}\right)$. In another study, a comparison was made between 261 knees replaced with Medial Pivot fixed-bearing prostheses and 288 replaced with 913 posterior-stabilized fixed-bearing prostheses (Wright Medical) [22]. There was no major difference in the flexion obtained 12 months after surgery $\left(111^{\circ}\right.$ and $109^{\circ}$, respectively). However, regression analysis of an individual knee revealed a small (average, $2.9^{\circ}$ ) but greater loss of flexion 12 months after surgery with the Medial Pivot fixed-bearing prosthesis. The authors concluded although the Medial Pivot fixed-bearing prosthesis may have improved kinematics, in terms of reduced paradoxical movement, it has no advantage in improving flexion at 12 months.

We found the mean ranges of knee motion were $124^{\circ}$ for the Medial Pivot fixed-bearing prosthesis before surgery and $115^{\circ}$ at final followup. For the PFC Sigma mobilebearing prosthesis, the mean ranges were $123^{\circ}$ before surgery and $127^{\circ}$ at final followup. The reason for loss of ROM with the Medial Pivot fixed-bearing prosthesis at final followup is unclear. We suspect intended restoration of the normal kinematics of the Medial Pivot fixed-bearing prosthesis might not be achieved to improve ROM of the knee.

Patient satisfaction and preference for fixed- and mobile-bearing prostheses were compared by numerous authors [16, 26, 27]. These authors reported negligible differences in patient satisfaction and preference between the two types of implants. In the series of PFC Sigma fixedand PFC Sigma mobile-bearing prostheses, patient satisfaction and preference were similar in both groups [15]. We found patient satisfaction higher with the PFC Sigma mobile-bearing prosthesis. We attributed dissatisfaction with the Medial Pivot fixed-bearing prosthesis to a higher incidence of infection and insufficient ROM.

Incidence of infection after TKA has decreased during the past few decades because of improvements in prevention efforts and surgical techniques. The most notable improvement occurred with the routine use of perioperative prophylactic antibiotics. Currently, the risk for postoperative infection after TKA is approximately $0.4 \%$ to $2 \%$ $[9,10,19]$. Risk factors include patient or host variables, surgical technique, and various aspects regarding surgical environment and postoperative management of the patient [9]. The causes of the high incidence of superficial infection $(3.3 \%)$ and deep infection $(2.7 \%)$ in knees with the Medial Pivot fixed-bearing prosthesis in our series is unclear. No patient in either group had specific risk factors. One experienced knee surgeon performed all TKAs in this series. Therefore, the surgeon-specific factor would not be a contributing factor to the high incidence of infection. We suspect failure in restoration of normal kinematics with the Medial Pivot fixed-bearing prosthesis might lead to irritation of soft tissues and subsequently recurrent effusion and infection in the knee.

Although the design features of the Medial Pivot fixedbearing prosthesis reportedly improve kinematics compared with those of previous TKAs using fixed-bearing prostheses, we found worse early clinical outcomes, less ROM, less patient satisfaction, and a higher complication rate in the patients with a Medial Pivot fixed-bearing prosthesis than in the patients with a PFC Sigma mobilebearing prosthesis. 
Acknowledgments We thank Sang-Mi Lee, BA, The Joint Replacement Center of Korea, Ewha Womans University DongDaeMun Hospital, for recording radiographic data and statistical analysis.

Open Access This article is distributed under the terms of the Creative Commons Attribution Noncommercial License which permits any noncommercial use, distribution, and reproduction in any medium, provided the original author(s) and source are credited.

\section{References}

1. Argenson JN, O'Connor JJ. Polyethylene wear in meniscal knee replacement: a one to nine-year retrieval analysis of the Oxford knee. J Bone Joint Surg Br. 1992;74:228-232.

2. Barrack RL, McClure JT, Burak CF, Clohisy JC, Parvizi J, Hozack W. Revision total hip arthroplasty: the patient's perspective. Clin Orthop Relat Res. 2006;453:173-177.

3. Bartel DL, Bicknell VL, Wright TM. The effect of conformity, thickness, and material on stresses in ultra-high molecular weight components for total joint replacement. J Bone Joint Surg Am. 1986;68:1041-1051.

4. Buechel FF Sr, Buechel FF Jr, Pappas MJ, D'Alessio J. Twentyyear evaluation of meniscal bearing and rotating platform knee replacements. Clin Orthop Relat Res. 2001;388:41-50.

5. Bugbee WD, Ammeen DJ, Parks NL, Engh GA. 4- to 10-year results with the anatomic modular total knee. Clin Orthop Relat Res. 1998;348:158-165.

6. Colizza WA, Insall JN, Scuderi GR. The posterior stabilized total knee prosthesis: assessment of polyethylene damage and osteolysis after a ten-year-minimum follow-up. J Bone Joint Surg Am. 1995; 77:1713-1720.

7. Diduch DR, Insall JN, Scott WN, Scuderi GR, Font-Rodriguez D. Total knee replacement in young, active patients: long-term follow-up and functional outcome. J Bone Joint Surg Am. 1997;79:575-582.

8. Engh GA. Failure of the polyethylene bearing surface of a total knee replacement within four years: a case report. J Bone Joint Surg Am. 1988;70:1093-1096.

9. Hanssen AD, Rand JA. Evaluation and treatment of infection at the site of a total hip or knee arthroplasty. Instr Course Lect. 1999;48:111-122.

10. Hill C, Flamant R, Mazas F, Evrard J. Prophylactic cefazolin versus placebo in total hip replacement: report of a multicentre double blind randomised trial. Lancet. 1981;1:795-796.

11. Hollister AM, Jatana S, Singh AK, Sullivan WW, Lupichuk AG. The axes of rotation of the knee. Clin Orthop Relat Res. 1993;290:259-268.
12. Insall JN, Dorr LD, Scott RD, Scott WN. Rationale of the Knee Society clinical rating system. Clin Orthop Relat Res. 1989; 248:13-14.

13. Insall JN, Hood RW, Flawn LB, Sullivan DJ. The total condylar knee prosthesis in gonarthrosis: a five to nine-year follow-up of the first one hundred consecutive replacements. J Bone Joint Surg Am. 1983;65:619-628.

14. Insall JN, Ranawat CS, Aglietti P, Shine J. A comparison of four models of total knee-replacement prostheses. J Bone Joint Surg Am. 1976;58:754-765.

15. Kim YH, Kim DY, Kim JS. Simultaneous mobile- and fixedbearing total knee replacement in the same patients: a prospective comparison of mid-term outcomes using a similar design of prosthesis. J Bone Joint Surg Br. 2007;89:904-910.

16. Kim YH, Kook HK, Kim JS. Comparison of fixed-bearing and mobile-bearing total knee arthroplasties. Clin Orthop Relat Res. 2001;392:101-115.

17. Menchetti PP, Walker PS. Mechanical evaluation of mobile bearing knees. Am J Knee Surg. 1997;10:73-81, discussion 81-82.

18. O'Connor JJ, Goodfellow JW. Theory and practice of meniscal knee replacement: designing against wear. Proc Inst Mech Eng [H]. 1996;210:217-222.

19. Peersman G, Laskin R, Davis J, Peterson M. Infection in total knee replacement: a retrospective review of 6489 total knee replacements. Clin Orthop Relat Res. 2001;392:15-23.

20. Schai PA, Thornhill TS, Scott RD. Total knee arthroplasty with the PFC system: results at a minimum of ten years and survivorship analysis. J Bone Joint Surg Br. 1998;80:850-858.

21. Schmidt R, Komistek RD, Blaha JD, Penenberg BL, Maloney WJ. Fluoroscopic analyses of cruciate-retaining and medial pivot knee implants. Clin Orthop Relat Res. 2003;410:139-147.

22. Shakespeare D, Kinzel V, Ledger M. Achieving ligament stability and correct rotational alignment of the femur in knee arthroplasty: a study using the Medial Pivot knee. Knee. 2005;12:419-423.

23. Stern SH, Insall JN. Posterior stabilized prosthesis: results after follow-up of nine to twelve years. J Bone Joint Surg Am. 1992;74:980-986.

24. Stiehl JB. Mobile bearings in total knee arthroplasty. In: Scott WN, ed. Insall \& Scott Surgery of Knee. Vol 2. Philadelphia, PA: Churchill Livingstone Elsevier; 2006:1588-1573.

25. Wohlrab D, Ditl J, Herrschelmann R, Schietsch U, Hein W, Hube R. [Does the NexGen LPS flex mobile knee prosthesis offer advantages compared to the NexGen LPS?: a comparison of clinical and radiographic results][in German]. Z Orthop Ihre Grenzgeb. 2005;143:567-572.

26. Woolson ST, Northrop GD. Mobile vs fixed-bearing total knee arthroplasty: a clinical and radiologic study. J Arthroplasty. 2004;19:135-140.

27. Wright TM, Bartel DL. The problem of surface damage in polyethylene total knee components. Clin Orthop Relat Res. 1986;205:67-74. 